InterSedes, Revista electrónica de las sedes regionales de la Universidad de Costa Rica, ISSN 2215-2458, Vol XXI, Número 43, Enero - Julio, 2020.

10.15517/isucr.v21i43.41990 | intersedes.ucr.ac.cr | intersedes@ucr.ac.cr

\title{
SINERGIAS EN LOS PROCESOS DE DESARROLLO RURAL DE UN PROYECTO DE COOPERACIÓN ENTRE JAPÓN Y COSTA RICA
}

\section{SYNERGIES IN THE RURAL DEVELOPMENT PROCESSES OF A COOPERATION PROJECT BETWEEN JAPAN AND COSTA RICA}

\author{
Carmen Monge-Hernández ${ }^{1}$ \\ Priscilla Mena-García ${ }^{2}$ \\ Marcela Gutiérrez-Miranda ${ }^{3}$ \\ Rita Gamboa-Conejo ${ }^{4}$ \\ Miguel Céspedes-Araya ${ }^{5}$
}

\begin{tabular}{ll} 
Recibido: 21.02 .19 & Aprobado: 30.04 .20 \\
\hline
\end{tabular}

DOI: $10.15517 /$ isucr.v21i43.41990

\section{Resumen}

A partir de las buenas prácticas entre universidades, instituciones públicas y organizaciones nacionales e internacionales en el ámbito del desarrollo rural en Costa Rica, emerge la necesidad de entender y visibilizar los retos y logros alcanzados desde la perspectiva de las personas participantes locales. Por ello, la presente investigación cualitativa de corte exploratorio propone analizar un proyecto de desarrollo rural realizado en el norte de Costa Rica, desde el enfoque de capacidades para el desarrollo humano. Tal experiencia llevada a cabo en, con y para la comunidad, destaca por su carácter transformador y por potenciar la ampliación de las capacidades individuales y colectivas de las comunidades, proponiendo desafíos atendibles para la sostenibilidad de las experiencias.

Palabras claves: desarrollo rural; enfoque de las capacidades; desarrollo humano; cooperación internacional.

\footnotetext{
${ }^{1}$ Costarricense. Docente Universidad Nacional de Costa Rica, Heredia, Costa Rica. Email: cmonge@una.cr

${ }^{2}$ Costarricense. Docente Universidad Nacional de Costa Rica, Heredia, Costa Rica. Email: pris.mena@gmail.com

${ }^{3}$ Costarricense. Docente Universidad Nacional de Costa Rica, Heredia, Costa Rica.

Email: marcela.gutierrez.miranda@una.cr

${ }^{4}$ Costarricense. Docente Universidad Nacional de Costa Rica, Heredia, Costa Rica. Email: rgamboa55@yaho.com

${ }^{5}$ Costarricense. Docente Universidad Nacional de Costa Rica, Heredia, Costa Rica. Email: macautos@yahoo.com
} 


\begin{abstract}
Based on good practices developed by universities, public institutions and national and international organizations that work on rural development in Costa Rica; it is needed to understand and make visible the faced challenges and achievements from the local participants perspective. The present exploratory qualitative research analyzes a rural development project carried out in the north of the country. From the capabilities approach for human development, the experience was carried out in, with and for the community. It stands out for its transforming nature, for enhancing the expansion of individual and collective capacities in the communities and for proposing challenges for its sustainability.
\end{abstract}

Keywords: rural development; capabilities approach; human development; international cooperation.

Generalidades de la cooperación en Costa Rica

En Costa Rica, país centroamericano, la cooperación internacional es canalizada a través de los Ministerios de Relaciones Exteriores y Culto y el de Planificación Nacional y Política Económica (MIDEPLAN). A MIDEPLAN le corresponde la negociación, el seguimiento a los proyectos y programas, el intercambio de experiencias y la oferta técnica que se presente, así como el manejo de los recursos financieros reembolsables y no reembolsables. En América Latina la tendencia de la Ayuda Oficial al Desarrollo (AOD) a partir de 1990 ha sido decreciente, lo que se ha acentuado aún más en países como Costa Rica, a los que organismos internacionales como el Banco Mundial (BM) y la Organización para la Cooperación y el Desarrollo (OCDE) les han catalogado como país de renta media.

La cooperación hacia los países en desarrollo ha transitado en las últimas décadas por distintos objetivos de reforma, modernización, estabilización y seguridad internacional (Montufar, 2004; Sogge, 2009, Kaul et al.,1999, Ayllon et. al., 2013). Adicionalmente, la influencia de nuevos eventos internacionales reorienta la cooperación. Tal es el caso de la búsqueda de reducción de los devastadores impactos de las crisis, en ámbitos alimentarios, energéticos, financieros internacionales y del calentamiento global y el atentado del 11 de setiembre 2001. Lo que alentó a los países desarrollados a buscar el aseguramiento de los suministros de combustibles fósiles para sostener su crecimiento desplazando la ayuda a la lucha contra el terrorismo internacional (Sanahuja, 2006). 
A partir de los noventa, la disminución de la ayuda a países de renta media abrió espacio a nuevos esquemas de cooperación al desarrollo. Esto ha permitido a Costa Rica realizar cooperación Sur-Sur y cooperación triangular, a pesar de no contar con fondos específicos para este fin. Ejemplo: los programas Sur-Sur con México, Chile, Uruguay, Argentina, Colombia y Perú y de triangulación con países de Centroamérica, el Caribe Alemania y España (MIDEPLAN, 2014a). Aunque los montos contabilizados son bajos, son valiosas en términos de intercambio de experiencias y asistencia técnica entre países.

Las convenciones internacionales han aportado impulso a cambios positivos en la cooperación, como por ejemplo la Declaración de París sobre la Eficacia de la Ayuda al Desarrollo (2005) y el Plan de Acción de Accra (2008) ${ }^{6}$. Esto favoreció la política exterior y la cooperación internacional costarricense (MIDEPLAN, 2014a) pues estimulan a los países receptores para que ejerzan una mayor autonomía y autoridad en sus políticas y estrategias de desarrollo a la hora de perseguir las metas globales para la reducción de la pobreza y la desigualdad.

En Costa Rica, entre 1990 y 2013 la AOD se ha reducido en un 78\%, pasando de 287.6 millones de dólares a apenas 63.3 millones de dólares. Según MIDEPLAN (2014b), entre 2010 y 2013 la cooperación no reembolsable se redujo en más de la mitad, con US\$144,2 millones en 2010 y US\$63,3 millones en 2013. Asimismo, si se considera la cifra total de cooperación al desarrollo durante el periodo 2010 y 2013, únicamente el 7,1\% de los US\$4.940,2 millones recibidos corresponde a flujos no reembolsables; el restante 92,2\% corresponde a la cooperación financiera reembolsable (MIDEPLAN, 2014a).

Si bien es una realidad que Costa Rica presenta mejoras importantes en los indicadores sociales y económicos, esta condición positiva no excluye la existencia de fragilidad ambiental, desigualdad y pobreza en zonas rurales, especialmente en las áreas fronterizas donde se asientan grupos altamente vulnerables. Por lo que se necesitan programas y proyectos públicos y apoyo

\footnotetext{
${ }^{6}$ La Declaración de París establece como principios fundamentales la armonización, apropiación, alineación, con la mutua responsabilidad oferente-beneficiario y gestión por resultados. Y con el interés de acelerar la aplicación de esa declaración se firma la siguiente declaración en Accra, Ghana, el 4 de septiembre de 2008 (https://www.oecd.org/dac/effectiveness/34580968.pdf, consultado el 15/03/2017).
} 
internacional para la financiación de iniciativas, principalmente en zonas fronterizas rurales y urbanas socialmente deprimidas.

La Universidad Nacional de Costa Rica (UNA), sustentada en la extensión universitaria, implementa acciones para el desarrollo rural, en las que convergen iniciativas financiadas con recursos de cooperación japonesa no reembolsables en comunidades rurales del norte y caribe noreste de Costa Rica, campo de esta investigación, para la que si bien abundan documentos e informes técnicos y económicos de la asignación de los recursos invertidos, hay carencia de literatura que valore los beneficios obtenidos desde la voz de las personas participantes. Por tanto, esta investigación exploratoria estudia un proyecto de cooperación asiática específico, a fin de ampliar la evaluación limitada a términos económicos de la relación donante, Estado y comunidad (centrada en el objeto), a una más cualitativa (centrada en los sujetos). Para esto se apoya en el enfoque de las capacidades para el desarrollo humano, desde una comprensión amplia del bienestar y la libertad de las personas para acceder a una vida digna (Walker, 2014).

El análisis cualitativo se realiza en el Proyecto para la promoción y el manejo participativo en la conservación de la biodiversidad (MAPCOBIO), desarrollado en Llanuras del Gaspar y Barra del Colorado, ubicados al noreste del país y financiado por Agencia de Cooperación Internacional del Japón (JICA). Interesa identificar los resultados alcanzados desde la voz de las personas locales participantes, en función de los aportes a su desarrollo humano.

Para mostrar los resultados de la investigación, se muestra en un primer apartado una presentación de las experiencias de estudio, seguido por los principales argumentos teóricos propuestos por el enfoque de las capacidades que guían el análisis. En el segundo apartado se hace referencia a los métodos utilizados y en el tercero se exponen los principales resultados obtenidos con el análisis y la discusión de la realidad local, identificándose algunas de las capacidades reales desarrolladas desde la perspectiva de las personas participantes. Finalmente, se presentan las conclusiones claves del estudio desde los resultados y los aprendizajes estratégicos que aporta esta experiencia japonesa al desarrollo local.

Cooperación de Japón con Costa Rica 
La cooperación internacional asiática ha mantenido apoyo a Costa Rica, mediante la cooperación de China, Japón y Corea. Los aportes reembolsables y no reembolsables del Gobiernos de la República de China han cambiado la composición de la cooperación internacional recibida por Costa Rica. Aunque la cooperación multilateral representó históricamente el 80\% de la cooperación, respecto a la bilateral, en el periodo 2010-2013 el flujo bilateral ha incrementado sustancialmente, acercándose a la fuente multilateral (Mideplan, 2014a). Para el periodo de 2009 - 2013, la cooperación bilateral proviene de Asia (93,1\%), Europa $(5,1 \%)$ y América $(1,8 \%)^{7}$ y los aportes no reembolsables ascienden alrededor de los 2300 millones de dólares ${ }^{8}$.

Los nexos de cooperación con Japón datan desde 1935, con más de 80 años de relaciones bilaterales, por medio de la Agencia de Cooperación Internacional de Japón (JICA). En los ejes prioritarios destacan la conservación ambiental y desarrollo sostenible, el fortalecimiento de la competitividad e innovación bienestar social, la seguridad social ${ }^{9}$. Su apoyo pretende la formación del recurso humano que contribuya en la mejora económica y social para un desarrollo integral de la sociedad. Se busca no solo realizar la transferencia tecnológica en áreas vitales sino alcanzar la meta de lograr que exista una comprensión mutua entre pueblos por medio del intercambio humano ${ }^{10}$. Se proponen impactar positivamente en los sectores beneficiarios e integración en los procesos de desarrollo social mediante proyectos de cooperación, con duración de 3 a 5 años. Estos proyectos ofrecen asistencia integral desde la fase de formulación, planificación, ejecución y evaluación con el apoyo de expertos, becarios, voluntariado y donación de equipos para generar las condiciones de sostenibilidad de los procesos puestos en marcha, especialmente con las comunidades de áreas rurales.

MAPCOBIO constituye un proyecto de ayuda técnica, no reembolsable, resultante de la cooperación antes aludida, ejecutadas en dos fases; la primera entre 2008 y 2011 y, la segunda, entre el 2013 y el 2018. Parte de las motivaciones de esta relación surgen con la finalización del

\footnotetext{
${ }^{7}$ Los flujos provienen específicamente de China (64,4\%), Japón (26,4\%), Alemania (3,8\%), Corea (2,3\%) y Estados Unidos, Holanda y España (1,9\%, 0,7\% y 0,4\% respectivamente) (MIDEPLAN, 2014b).

8 Otorgados por China (1445 millones), Japón (600 millones), Alemania (84,9 millones), Estados Unidos (58,9 millones), España (52 millones) y Corea (51,6 millones) (MIDEPLAN, 2014b).

${ }^{9}$ Tomado de http://www.cr.emb-japon.go.jp/coope/coope.html, consultado el 28/2/2017.

10 Tomado de http://www.cr.emb-japan.go.jp/coope/coope.html, consultado el 28/2/2017.
} 
apoyo en áreas ambientales por parte de la Unión Europea y el interés japonés en aportar a la conservación del ambiente desde una visión de desarrollo sostenible; aprovechando el marco jurídico costarricense para la implementación del manejo participativo en áreas silvestres protegidas.

La primera parte de MAPCOBIO buscó legitimar la tenencia de la tierra, estableciendo los territorios que le pertenecen al Estado en el humedal Caribe Noreste ${ }^{11}$, debido a que esta zona presentaba una gran irregularidad de la tenencia y tenía personas viviendo en humedales e islas propiedad del Estado. A partir de un mapa catastral de algunos sectores de la zona se detectó el traslape de propiedades y delimitó los territorios que pertenecen al Estado. Esto aportó al fortalecimiento de las instituciones nacionales con la contratación de personal por parte de JICA y de Ministerio de Ambiente y Energía (MINAE) ${ }^{12}$. Esos nuevos funcionarios fueron capacitados por la administración del Refugio de Vida Silvestre Barra del Colorado para encargarse del proceso en esa área protegida. Durante esta etapa se identificaron y fortalecieron 150 experiencias de manejo participativo en el territorio nacional, con el objetivo de dar seguimiento en la quinta etapa, se seleccionaron 22 experiencias.

La iniciativa 2013 - 2018 tiene como objetivo aportar a la conservación de los recursos con la gente. Para esto se enlazan acciones interinstitucionales con el Ministerio de Educación Pública (MEP), el Ministerio de Agricultura y Ganadería (MAG) y el Instituto de Desarrollo Rural (INDER) para lograr incidir de manera asertiva en el bienestar de la población. Como parte de los productos, el Sistema Nacional de Áreas de Conservación (SINAC) y JICA elabora un Plan General de Manejo para el Humedal de importancia internacional Caribe Noreste. Este plan facilitó identificar deficiencias en la estructura organizativa en dos comunidades: Linda Vista y Llanuras del Gaspar como condición limitante para las oportunidades de desarrollo y la conservación de los recursos naturales de la zona.

\footnotetext{
${ }^{11}$ No obstante, MAPCOBIO fue ejecutada a nivel nacional, debido a que en el país existe una dispersa y abundante participación ciudadana en temas de conservación de la biodiversidad. La iniciativa buscó unificar esfuerzos para mejorar la conservación.

${ }^{12}$ MINAE se comprometió a la contratación funcionarios para la ejecución de la iniciativa, debido a que JICA no otorga fondos para la contratación permanente de personal.
} 
Por tal razón, MAPCOBIO, desarrolló un proceso de fortalecimiento y construcción de una base organizativa sólida que permita mejorar las oportunidades de los pobladores de estas dos zonas, que se analizan en esta investigación. A partir del 2015 se conforman asociaciones locales en las comunidades con el fin de generar y mejorar oportunidades de empleo en la zona desde una visión de desarrollo con conservación ambiental. Estas asociaciones deciden trabajar, una en el tema de turismo rural comunitario y la otra en la elaboración y comercialización de quesos. Además, se trabaja en el manejo participativo de las áreas de conservación a nivel intersectorial, como método de fortalecimiento de la iniciativa.

No obstante, las bondades del proyecto en el plano operativo, se requiere fundamentar sobre bases científicas el desarrollo humano, necesario para el aprovechamiento de los recursos naturales, en función de una organización social más activa y armónica con la naturaleza.

El enfoque de las capacidades para el desarrollo humano como marco orientador de análisis

La caracterización anterior de MAPCOBIO pareció a las autoras un campo ideal de desarrollo humano que sustenta esta investigación basada en la noción de desarrollo humano, inspirada en los setentas por Amartya Sen y otros propulsores, a partir de la necesidad de centrar las acciones de desarrollo y bienestar en el ser humano; considerando a las personas como medio y fin del desarrollo (Unceta y Koldi, 2000, Sen, 2000). El desarrollo humano se basa en la idea de ampliar las opciones de desarrollo de las personas y aunque esas opciones pueden resultar infinitas y variar en el tiempo, las valoraciones de las personas se focalizan en áreas para su desarrollo, como son el acceso al conocimiento, una mejor nutrición, acceso a servicios de salud, seguridad, tiempo libre de calidad, participación y libertades políticas, entre otras. Es decir, en aspectos que no solo están vinculados a los ingresos (U1 Haq1995).

Esta perspectiva ha permitido trascender la visión economicista, en donde el crecimiento económico figura como un fin en sí mismo, con gran peso en la toma de decisiones, hacia una visión de desarrollo social, humano. El desarrollo humano es el proceso mediante el que se amplían las oportunidades de las personas "entendiendo que dichas oportunidades dependen en lo fundamental de las capacidades y libertades de las que puedan disponer, y del papel que ellas 
mismas tengan como protagonistas del desarrollo" (Unceta y Koldi, 2000, p, 82). Esto plantea profundos retos para la asistencia social y la cooperación al desarrollo, para lograr contribuciones reales de avance al desarrollo humano de grupos socialmente vulnerables.

La idea de desarrollo humano se difunde a partir de los años noventa, mediante el Programa de las Naciones Unidas para el Desarrollo (PNUD); el Informe sobre desarrollo humano (IDH) de 1990 que integra aspectos socio ambientales sobre la base de que "la verdadera riqueza de la nación está en su gente" (PNUD, 1990, p. 31). En estos informes se supera el énfasis económico de los estudios de desarrollo e incorpora otros ámbitos como la educación, la salud y la satisfacción de las necesidades básicas. Según PNUD (1990), el desarrollo humano es un proceso que permite que se amplíen las oportunidades de las personas para "una vida prolongada y saludable, acceso a la educación y el disfrute de un nivel de vida decente" (p. 33). Esto implica lograr que, "las personas puedan desarrollar su máximo potencial y llevar adelante una vida productiva y creativa de acuerdo con sus necesidades e intereses [...] que cada persona pueda vivir una vida que valore" (PNUD, 2017, p.1).

El enfoque de capacidades se ha consolidado como un marco normativo que favorece la evaluación del bienestar individual, de propuestas para el cambio social, además de emitir juicios y abordar integralmente aspectos vinculados al desarrollo, bienestar y justicia social (Robeyns, 2003). Al respecto Sen (2000) aporta los conceptos clave de capacidades, funcionamientos y agencia, donde el primero se relaciona con la libertad de las personas u oportunidades para lograr funcionamientos. Las capacidades son combinaciones de funcionamientos que puede lograr una persona (seres y haceres) y son el reflejo de la libertad de una persona para llevar la vida que valoran (Alkire, 2002, pp.181-205). La agencia comprende la habilidad de una persona para definir de manera autónoma sus metas propias y llevarlas a la práctica, es "aquello que una persona tiene la libertad de hacer y lograr en búsqueda de las metas o valores que él o ella considere importantes" (Sen, 1985, p. 203).

El análisis de las políticas o procesos de cooperación al desarrollo, pertinente a esta investigación, incluye las barreras que les impide a los participantes ampliar sus capacidades y aprovechar sus libertades (Robyens, 2013). Por tanto, hay que entender los factores que influyen 
en el resultado que logran las personas con la iniciativa. Para esto hay que analizar los factores de conversión personal (condición física y psicológica), institucional (marco político, institucional) y medioambiental (condiciones ambientales) que interfieren para que las personas puedan convertir las oportunidades (o libertades) en funcionamientos reales (Robyens, 2013).

Metodología de investigación

A partir de la contradicción entre las visiones economicista y social-participativa de los proyectos, la primera centrada en el capital material y financiero y la última en el desarrollo humano (sin detrimento de la efectividad del proyecto en otros órdenes económicos), se centra el problema del presente trabajo. En correspondencia con tal contradicción y la actitud impugnante de las autoras del modelo economicista, el objetivo de esta investigación es fundamentar el proyecto de ayuda técnica MAPCOBIO en el enfoque de capacidades para el desarrollo humano, tomando en cuenta la contribución que realizan los procesos de cooperación al desarrollo humano en las personas participantes, así como las principales barreras que limitan el impacto del proceso desarrollado en la comunidad.

La fundamentación toma como argumento los resultados del análisis de la aplicación de metodologías cualitativas que corresponde a "un conjunto de prácticas interpretativas que hacen el mundo visible" (Denzin y Lincoln, 2005, p. 3), lo que permite el registro de las perspectivas de las personas que han estado implicadas en las experiencias de cooperación seleccionadas.

La investigación se desarrolla en tres fases: la primera es de revisión documental (propuestas, informes, expedientes de proyectos y normativa institucional, en su mayoría no publicados). La segunda fase consiste en la recolección de datos primarios, mediante entrevistas semiestructuradas, grupos de discusión y la observación participante en campo (Corbetta, 2007). La observación participante se realizó mediante diversos recorridos por los proyecto de turismo rural comunitario y el fortalecimiento a las fincas vinculadas con la producción de leche y queso para conversar directamente con las personas participantes y constatar los avances alcanzados. Las entrevistas se aplicaron a: 6 funcionarios de las instituciones nacionales y de la agencia de cooperación y a 8 beneficiarios locales; así mismo, se desarrolló un grupo de discusión con 10 
beneficiarios. En la última etapa, se realiza la triangulación de la información obtenida de las diferentes fuentes y se analizan los principales hallazgos.

Análisis de resultados y discusión

Este apartado se construye tomando en cuenta lo que las personas más valoran del proceso desarrollado por MAPCOBIO partiendo de asumir a los participantes locales como protagonistas en la identificación de sus logros.

\section{Población}

Para las personas locales las acciones de formación, asesoría y capacitaciones han sido prioritarias y estratégicas para iniciar sus cambios personales, la generación de proyectos socioproductivos y concebir un desarrollo conservando el ambiente. Sus esfuerzos están motivados por la superación de limitantes para sostener sus fincas y generar ingresos que les permita sostener sus costos de vida y permitir que sus familias bien mejor.

Parte de los logros generados a partir de MAPCOBIO, y que genera reconocimiento social y expectativas para las personas participantes, es concretamente la conformación de dos asociaciones en zonas que tienen profundas limitaciones socio-económicas, la Asociación Linda Vista (ASOLIVI) y la Asociación de Productores de Llanuras del Gaspar (ASOPRO).

ASOLIVI nace en el 2015, en pro-bienestar social de la comunidad de Linda Vista, integrada por doce personas de la comunidad ${ }^{13}$. Esta comunidad posee condiciones de difícil acceso a los servicios de agua potable, electricidad y telecomunicaciones. La comunidad de Linda Vista reconoce que JICA permitió un proceso muy provechoso para las personas, con ASOLIVI se unió la conservación del ambiente y el desarrollo local, que atiende el espacio territorial respetando la normativa de refugio de vida silvestre mixto ${ }^{14}$. A partir del proceso realizado se logró entender que la geografía y la riqueza en biodiversidad le conceden gran potencial al turismo rural comunitario, por lo que esta nueva organización se orienta a desarrollar proyectos

${ }^{13}$ La comunidad se ubica en el cantón de Pococí, provincia de Limón, en el Área silvestre protegida Refugio Nacional de Vida Silvestre Barra del Colorado (MIVAH, 2012). Se fundó en 1982 y está formada por 36 familias, de las cuales 9 de ellas integran ASOLIVI.

${ }^{14} \mathrm{Al}$ ser un refugio de vida silvestre mixto la normativa determina que las acciones de conservación se deben hacer en conjunto con las comunidades inmersas (Calvo y Marín, 2014). 
locales que reactiven la economía local e impacten socialmente a la comunidad, pero que, a la vez, pueden desarrollarse en armonía con la naturaleza.

En la iniciativa de turismo rural comunitario se desarrolla la Ruta de los Aventureros Alas y Raíces; que ofrece la experiencia de contacto directo con la flora y fauna de las montañas del Caribe costarricense y de interactuar con comunidad preocupada por preservar el ambiente como herramienta para asegurar su bienestar y el de sus familias. Los y los asociados, en las entrevistas, permiten dar a conocer su satisfacción e importancia de la cooperación japonesa, clave para la conformación, el fortalecimiento y el desarrollo de la organización.

Por su parte ASOPRO está ubicada Sarapiquí de Heredia ${ }^{15}$, donde las principales preocupaciones son el empleo, la infraestructura vial, la organización colectiva para la comercialización de la producción local y la coordinación interinstitucional (Montes y Matamorros, 2011). ASOPRO busca principalmente la comercialización de los derivados de la leche y lograr que las fincas sean manejadas sosteniblemente por cada una de las familias (S. Calvo, comunicación personal, 23 de enero 2017).

La experiencia japonesa supera los resultados de uso de los instrumentos de asistencia técnica o transferencia tecnológica de la cooperación internacional cuyo objetivo se limitan a proporcionar conocimientos y habilidades técnicas y de gestión cualificando a los recursos humanos del receptor (Boni et. al. 2010). Los procesos logran ir más allá, a áreas de educación para el desarrollo e incidencia y presión política en el ámbito local; pues desarrollan un proceso educativo que favorece la comprensión de las interrelaciones entre actores de diversos ámbitos y la promoción valores y actitudes hacia un desarrollo humano sostenible.

Si bien se denota una similitud en la metodología de abordaje para el fortalecimiento de las capacidades de ASOPRO como ASOLIVI en ambas se ejecutaron actividades diferenciadas, ajustadas a la naturaleza del proyecto y del eje productivo y de servicios: turismo rural y producción de queso.

\footnotetext{
${ }^{15} \mathrm{El}$ distrito posee aproximadamente 1160 habitantes, 514 mujeres y 646 hombres. El índice de desarrollo social (IDS) de Llanuras del Gaspar presenta la posición 480 de los 483 distritos del país (MIDEPLAN, 2017).
} 
Los resultados obtenidos giran en torno al proceso de formación, asesoría y acompañamiento recibido durante y como resultado final del proyecto (Cuadro 1). Este resultado es crucial si se contempla que JICA se propuso impulsar el desarrollo aprovechando la amplia riqueza en recursos naturales existentes, pero conscientes de la necesidad de disponer de una organización social más activa que tuviera la capacidad de lograr un desarrollo en armonía con la naturaleza (A. Camacho, comunicación personal, 7 febrero 2017).

Cuadro 1. Acciones organizacionales del Proyecto MAPCOBIO-Costa Rica

\begin{tabular}{|l|c|c|}
\hline Áreas de formación, asesoría y acompañamiento & ASOPRO & ASOLIVI \\
\hline $\begin{array}{l}\text { Formación técnica: } \\
\text { Conocimientos técnicos } \\
\text { Biología, guías turísticas, atención al cliente e intercambio con } \\
\text { otros proyectos }\end{array}$ & $\mathrm{x}$ \\
\hline Invernaderos, cultivo de hortalizas & $\mathrm{x}$ & $\mathrm{x}$ \\
\hline $\begin{array}{l}\text { Manejo de residuos sólidos y de materiales subutilizados, } \\
\text { ganadería sostenible, mejoramiento genético y nutrición del hato } \\
\text { lechero }\end{array}$ & $\mathrm{x}$ & \\
\hline $\begin{array}{l}\text { Comercialización } \\
\text { Desarrollo de la ruta turística y signos externos }\end{array}$ & $\mathrm{x}$ \\
\hline Elaboración y comercialización del queso & $\mathrm{x}$ \\
\hline $\begin{array}{l}\text { Capacidades de gestión } \\
\text { Alianzas estratégicas (instituciones públicas, tour operadores) y } \\
\text { acompañamiento legal }\end{array}$ & & \\
\hline Administración y desarrollo de negocios & $\mathrm{x}$ & $\mathrm{x}$ \\
\hline $\begin{array}{l}\text { Fortalecimiento organizacional, propuestas para búsqueda de } \\
\text { recursos, alianzas institucionales para otras capacitaciones }\end{array}$ & & \\
\hline
\end{tabular}

Fuente: Elaboración propia con base en López et. al., 2016.

Sobre la expansión de capacidades valoradas por las personas participantes

En el Cuadro 2 se presentan algunas de las evidencias vinculadas a los logros de los participantes en áreas vinculadas a la motivación para la superación personal, el acceso a procesos formativos, las habilidades comunicativas y colaborativas, valores humanos $\mathrm{y}$ ambientales y la conexión con las instituciones para el apoyo financiero y el acceso a programas sociales. El común es encontrar que las personas valoran que hoy disponen de conocimientos nuevos que les permite atender mejor su finca, sus proyectos comunitarios y familiares.

Cuadro 2. Algunos de los logros alcanzados que más valoran las personas participantes locales

\begin{tabular}{|l|l}
\hline Logros o funcionamientos & Evidencias \\
\hline
\end{tabular}


Mayor comunicación, acercamiento y atención por parte de las y los funcionarios del sector público
"SENASA no venía antes a la zona, ahora nos capacita" (Entrevista 1, líder local); "El mayor beneficio es la capacitación que hemos recibido del INA [Instituto Nacional de Aprendizaje]" (Entrevista 1, líder local); "A partir de este proyecto, la relación con el MINAE ha mejorado" (Entrevista 2, líder local)

Apoyo financiero para proyectos de inversión

"El proyecto ha dado biodigestores a los finqueros y eso nos ha ayudado a provechar los recursos" (Entrevista 3, lideresa local)

\begin{tabular}{llll|l} 
Acceso a posibilidades de & El mayor beneficio es la capacitación que hemos
\end{tabular} capacitación en temas que necesitan

recibido del INA [Instituto Nacional de Aprendizaje]" (Entrevista 1, líder local); ASOPRO logró contar con un módulo de capacitación por parte de SENASA en ganadería sostenible y la práctica en elaboración y degustación de quesos

Acceder a equipos de investigación y lograr participar en el desarrollo de estudios valiosos para sus proyectos comunitarios y socio productivos

Adquisición de conocimientos que pueden aplicar en sus fincas

Tener una comunidad más
organizada

Logros diversos en lo personal, comunal y familiar.

"uno de los beneficios que valoramos es que nos ayudaron para elaborar un estudio de mercado sobre el queso" (Entrevista 1, líder local).

"he aprendido mucho, para mejorar mi finca, es más productiva ahora" (Entrevista 6, líder local)

"la comunidad está más organizada" (Entrevista 6 , líder local)

"hemos tenido muchos beneficios en todo nivel, personal, comunal y familiar" (Entrevista 7, líder local).

\begin{tabular}{|c|c|}
\hline $\begin{array}{l}\text { Tener intercambio cultural } \mathrm{y} \\
\text { aprender de otras personas }\end{array}$ & $\begin{array}{l}\text { Destacan haber conocido nuevas formas de } \\
\text { trabajo, aprender de otros y enriquecer su visión } \\
\text { de mundo (Entrevista } 1 \text { y } 2 \text {, lideresas locales). }\end{array}$ \\
\hline Empoderamiento femenino & $\begin{array}{l}\text { "a mí me ha impulsado a salir adelante, este } \\
\text { proceso con el proyecto me ayudó a salir de la } \\
\text { depresión, me ha ayudado a valorar que la vida } \\
\text { no termina con una separación" (Entrevista 1, } \\
\text { lideresa local). }\end{array}$ \\
\hline $\begin{array}{l}\text { Mayores lazos de amistad y } \\
\text { promoción de valores sociales y de } \\
\text { identidad comunitaria }\end{array}$ & $\begin{array}{l}\text { En los grupos de discusión reconocen que poseen } \\
\text { mayores lazos de amistad y más compromiso, } \\
\text { solidaridad, respeto al ambiente, responsabilidad, } \\
\text { confianza, permanencia, identidad y trabajo } \\
\text { colectivo. }\end{array}$ \\
\hline $\begin{array}{l}\text { Mayor capacidad de toma de } \\
\begin{array}{l}\text { decisiones, } \\
\text { participación y y } \\
\text { colectiva }\end{array}\end{array}$ & $\begin{array}{l}\text { "Estamos claros que JICA no nos va a dar dinero, } \\
\text { pero lo que necesitamos es acompañamiento para } \\
\text { llevar a cabo las acciones e ir a las instituciones } \\
\text { [nacionales] para que nos escuchen" (Entrevista }\end{array}$ \\
\hline
\end{tabular}


2, Lideresa local); Conformación de Juntas Directivas, comité pro centro de acopio para el reciclaje, construcción de senderos y jardín botánico, planes de trabajo, nuevos proyectos, atención de actividades varias en un mismo periodo.

Fuente: Elaboración propia a partir de los métodos aplicados.

Valoraciones cualitativas de los resultados

Para las personas locales el acceso a la cooperación japonesa es de partida la oportunidad más valorada por los actores locales. Esto no solo en función de las oportunidades de creación de capacidades necesarias para su desenvolvimiento, la credibilidad ante las instituciones del Estado y lograr concretar sus iniciativas socio- productivas y ambientales sino, además han logrado acceder colaboraciones adicionales. Por ejemplo, para la construcción de biodigestores e investigación de mercado de queso. Reconocen que sin esta ayuda su realidad actual sería muy distinta.

El compromiso con los procesos de formación desarrollados por JICA y la conexión de las organizaciones con las instituciones públicas ha permitido el avance de los proyectos socio productivos asociativos y que las personas accedan a conocimientos valiosos para su desarrollo individual y la gestión de sus fincas. ASOPRO logró contar con capacitaciones en temas prácticos como la producción de abono orgánico, mejor manejo de la finca, de salud animal, de manejo y prevención de suelos, entre otros. Además de un módulo de capacitación por parte de SENASA en ganadería sostenible y la práctica en elaboración y degustación de quesos. Estas capacitaciones permiten que las familias de ASOPRO puedan aspirar a llevar directo al mercado los productos, cumpliendo con la calidad y las medidas fitosanitarias necesarias. El ingreso al mercado de productos lácteos es una ventaja para los productores, al lograr mejores precios por sus productos, accediendo directamente al cliente final, sin intermediarios.

Hay incrementos en la apertura y las habilidades de comunicación y acercamiento efectivo con las instituciones públicas es fundamental para las personas que viven en estos contextos, especialmente por estar tan alejadas del área central del país y con pocas herramientas para acceder al poder político. Las personas entrevistadas reconocen que previo al apoyo de la cooperación japonesa no lograban captar la atención por parte de las organizaciones y se sentían 
olvidados y desprotegidos por la política pública. Este apoyo de la cooperación les ha permitido que se logre la integración con distintas instituciones para incrementar las capacidades técnicas para el desarrollo de la actividad.

Acompañado del incremento de capacidades técnicas y de gestión se ha logrado una mayor capacidad de planificación, participación y toma de decisiones. Estos cambios se expresan en las acciones realizadas por ASOPRO ante el Servicio Nacional de Salud Animal (SENASA) para lograr cumplir requisitos para comercializar el queso. Una mayor toma de decisiones y compromisos reflejada también procesos de planificación debidamente elaborada y consensuada entre asociados. Por ejemplo, plan de trabajo de ASOLIVI en turismo rural comunitario que integra actividades de generación de recursos y la inversión en protección y mejoras de sus condiciones de vida. Para su ejecución cuentan con recomendaciones de supervisión y ejecución del proyecto de turismo por parte de la Junta Directiva, medidas para potenciar el desarrollo efectivo del proyecto y sentar responsabilidad en todas las personas que integran la asociación.

La mayor capacidad de planificación, participación y toma de decisiones también se manifiesta en ASOLIVI, cuando de manera paralela al componente organizativo y de la producción hortícola se avanza en la ruta turística y la vinculación con organizaciones públicas y privadas. Este proyecto lo valoran importante porque les permite obtener ingresos sin dañar la naturaleza, conservando y cuidando la flora y fauna pueden generar ingresos para conseguir vivir tranquilos en sus hogares. Se trabaja en gestar alianzas estratégicas para su fortalecimiento a nivel empresarial y conectar a los turistas con la comunidad. El posicionamiento de la zona en la esfera turística nacional, genera otras opciones laborales y de recreación que potencian libertades fundamentales para quienes viven en la comunidad.

Durante la observación participante y los grupos de discusión se constató que las personas participantes se sienten satisfechas y motivadas con las acciones y los logros alcanzados. Esto ha favorecido la participación, el empoderamiento, la identificación de las personas con los procesos y apropiación de las acciones por parte de las partes. Importante destacar que se denotan también cambios en el empoderamiento de la mujer y en la forma en que los hombres propician su participación en la toma de decisiones. 
En lo cultural hay cambios, a nivel individual y colectivo en su apertura y actitud respecto a dedicar tiempo de su jornada diaria a las capacitaciones y trabajar en equipo. Reconocen que han logrado comprender que no se trata de un sacrificio, sino que es una inversión que determina los beneficios que han logrado obtener y que deben ir cambiando la lógica de enfocarse a su finca todo el día y dejar de lado las capacitaciones, porque eso les restringe radicalmente la posibilidad de cambiar su realidad a futuro. Durante las capacitaciones no solo aprenden de quien dicta el curso sino también del intercambio entre las y los productores y compañeros. Esto permite conocer los intereses y aspiraciones comunes, y les une y empodera a trabajar y luchar juntos. Además, han compartido salidas a otros poblados del país, con experiencias diferentes y aprendizajes durante visitas guiadas, como es el caso de la visita realizada a la Universidad EARTH y a Upala.

Otros componentes que representan buenas prácticas de la cooperación en el desarrollo rural se relaciona con cambios integrales en aspectos que mejoran la calidad de vida en las personas. Por ejemplo, con el cultivo de hortaliza, que no solo representan una oportunidad para atender clientes, sino también para mejorar su dieta diaria. O en el ámbito de la salud, con la gestión de desechos y el reciclaje a partir centro de acopio.

Hay valoraciones positivas en el crecimiento personal, asociadas a los conocimientos obtenidos, lazos entre vecinos y productores y de los avances en el desarrollo local y socio productivo de sus fincas, según las expresiones de los líderes del proyecto o productores. Las capacidades locales previas a iniciar proyectos colectivos en las comunidades rurales costarricenses son una condición adversa vigente. De acuerdo con las experiencias de extensión universitaria desarrolladas en otros contextos, hay que partir de la comprensión de que el desarrollo de un proyecto de desarrollo rural con comunidades sin antecedentes organizativos ni capacidades técnicas es muy complejo y requiere de un trabajo de planificación previa, adicional a las capacitaciones.

En el tema de inversión las comunidades presentan restricciones, pues el desarrollo de proyectos asociativos exige disponer de infraestructura y equipos de uso colectivo. ASOPRO con 
apoyo de la cooperación japonesa logró la instalación de tres estructuras de las cinco que habían planificado durante la ejecución del proyecto. Esta construcción permite disponer de las condiciones básicas para la venta de al menos 20 variedades diferentes de queso producido por diferentes socios y comercializado por la asociación. Para el cultivo de hortalizas, también fue necesario la instalación de sistemas de riego, la adquisición de otras técnicas agrícolas, visitas a cada uno de las familias participantes en su unidad productiva para instalar los sistemas y enseñar a elaborar bio-fermentos. Asimismo, dar el seguimiento a los cultivos de hortalizas, al desarrollo de cosechas y posterior resiembra de las áreas de cultivo.

En las entrevistas realizadas llama la atención que la totalidad de los participantes consideran que toda la comunidad se ha beneficiado de una u otra manera principalmente porque hoy viven en una comunidad organizada y perciben los beneficios subyacentes. Durante las entrevistas se puede notar evidencias de empoderamiento, liderazgo comunal y convicción de cambio, que se podrían asociar a lo que Amartya Sen denomina como agencia. Se trata de personas capaces de ejercer un liderazgo más comprometido con su comunidad, dispuestos a participar y aportar más a que se analicen y realicen proyectos que ayuden a la comunidad.

Algunos factores limitantes para el aprovechamiento de los procesos rurales

La narrativa de las personas participantes en las iniciativas locales denota un mayor peso y fuerte conexión entre dos grupos de factores, a saber: los factores sociales (institucionales) y los personales (de cada productor o actor local), lo que a su vez subsumen a otros inherentes. A continuación, se explican los mismos a partir de los registros obtenidos.

Hay factores socio-institucionales que restringen las libertades $\mathrm{u}$ oportunidades para aprovechar los procesos desarrollados por parte de las personas. Las pocas capacidades organizativas y de cohesión colectiva, la desconfianza, el temor a participar, tomar decisiones y planificar es parte de una cultura local que limita inicialmente las acciones. Esto, aunado a la carencia de conocimientos técnicos y habilidades organizativas y de gestión, reduce la posibilidad de que las personas logren liderar procesos de desarrollo rural desde ellos mismos. 
A lo anterior se suma factores personales, las personas participantes tienen una escolaridad de primaria o secundaria incompleta en la mayoría de los casos. Personas que reconocen que sin los conocimientos y colaboración para los estudios técnicos es prácticamente imposible generar proyectos comunitarios y productivos que permitan propiciar mejores en la comunidad y las familias. Necesitan encontrar alguna actividad productiva y penetrar en los mercados que permita los ingresos básicos estables para sostener las familias.

Las restricciones en materia de gestión organizacional es una variable constante de preocupación en el discurso de las y los pobladores locales. Consideran que, si bien que cada productor individualmente y como comunidad tienen claridad de sus necesidades prioritarias, les hace falta capacidades para sostener las ideas y ejecutar proyectos beneficiosos para todos. Esto lleva a demandar más asesoramiento en el planteamiento y ejecución de las acciones de manera más continua y en periodos más extensos. Como un escenario ideal, incluyen también en los procesos de desarrollo rural la dotación de apoyo financiero por parte de otras instituciones que les permita aplicar los conocimientos adquiridos y mejorar las fincas a partir de las capacitaciones obtenidas, por ejemplo, disponibilidad de asesoría técnica, acciones de seguimiento y oportunidades de créditos blandos, accesibles. Esto porque carecen de recursos y conocimiento técnicos para llevar los conocimientos a la práctica de manera inmediata, lo que les impide aprovechar rápidamente lo que van aprendiendo.

La cultura local y la dinámica de trabajo en sus fincas dificultan la participación y la disponibilidad de tiempo para dedicarse a la gestión, asistir a las capacitaciones y cumplir compromisos colectivos. No existe una cultura de formación que asegure la asistencia a capacitaciones. En estas comunidades rurales, las personas están acostumbradas a dedicarse a las actividades de su finca la mayor parte de su tiempo y consideran una pérdida dejar esas labores para atender las actividades comunales o del proyecto. Esto, además de propiciar metodologías que motiven la participación también demanda de realizar una planificación de trabajo con las comunidades en los horarios que a las personas locales más les convenga.

Las personas locales quisieran ver resultados en más corto tiempo y aunque intentan comprender que los procesos sociales de desarrollo son lentos, piden que se mejoren los 
esfuerzos por lograr procesos más efectivos y de mayor plazo. Su aspiración es ver resultados en la comunidad en menor plazo y con sostenibilidad de las iniciativas de largo plazo. Identifican debilidades en el seguimiento técnico a las iniciativas asociativas, que presionan a bajas en la motivación local y la efectividad de los resultados. Asimismo, exponen que "algunos de los consultores no son lo suficientemente buenos, a veces vienen urgidos por el tiempo y no nos atienden como debe ser" (Entrevista 3, lideresa local).

A pesar de la gran cantidad de capacitaciones recibidas consideran que necesitan adquirir mayores aprendizajes y que el intercambio de experiencias les ayuda en gran medida. Es parte de los factores que más valoran porque les aporta a comprender el funcionamiento de los procesos y la forma en que otros realizan sus actividades en lo cotidiano.

Según las personas de las comunidades consultadas, el plazo, los procesos de desarrollo rural impulsados desde organizaciones públicas y de este tipo de acciones de JICA es una variable negativa, pues es muy corto. Las personas aspiran a procesos de largo plazo que permitan apoyo en las diferentes fases del proyecto, incluyendo el acompañamiento en el diseño, planeación, ejecución, identificación de nuevos proyectos de desarrollo, asesoría, monitoreo hasta el seguimiento progresivo y permanente por parte de los técnicos. La comunidad alerta sobre la necesidad de que los consultores posean un compromiso real con el proyecto y con todas las personas de la comunidad con las que trabajan. Algunas personas sienten que los técnicos están más preocupados por cumplir sus contratos y recibir su pago que en entregarse realmente a la comunidad y dar un acompañamiento responsable, con visión de largo plazo.

Una limitante personal es que no logran cumplir con las condiciones que demandan las iniciativas colectivas derivadas del proceso desarrollado. Por ejemplo, aunque se ha logrado un gran avance en la organización y el mejoramiento de la comercialización del queso tienen el fuerte problema de no disponer de infraestructura (centro de acopio) de uso colectivo (líder 5). Esto frena las posibilidades de comercializar formalmente los productos de manera colectiva y desencadena descontento y desacuerdos entre productores. Esto les hace dudar de la existencia de mercado para sus productos o se tiende a desvalor los esfuerzos, aunque los logros alcanzados hayan sido sumamente positivos. 


\section{Reflexiones finales}

I Como lo plantea Sen, las condiciones de las personas en las comunidades no dependen solo de las decisiones individuales y colectivas que se gestan en esos territorios, sino también están determinadas por las acciones de apoyo social (Sen, 1999). Por tanto, y como deja evidencia esta investigación, comunidades con características similares a estos contextos analizados demandan de procesos de desarrollo rural, desde las distintas universidades públicas y de las demás instituciones del Estado y organizaciones de apoyo social nacionales e internacionales.

El abordaje del Proyecto MAPCOBIO, realizado con recursos de cooperación japonesa, tiene características de integralidad importantes, que abarca aspectos que impactan en lo personal, ambiental, económico y cultural de las personas participantes. El trabajo se ha centrado en dar herramientas a las personas, en capacidades vitales para su desarrollo en armonía con el ambiente. Este tipo de capacidades permite a las personas obtener logros (o funcionamientos) y elevan su nivel de agencia. Gracias al levantamiento de sus capacidades técnicas y humanas se han logrado resultados positivos importantes en lo personal, principalmente derivados del proceso formativo y la creación de capacidades organizativas (empoderamiento, participación, gestión, planificación de acciones, trabajo colectivo, entre otras). Se logró la conformación de dos organizaciones comunales, todo un encadenamiento de motivaciones en torno a las iniciativas. Hoy las personas participantes consultadas reconocen un incremento en su consciencia ambiental, en el compromiso con el desarrollo de su comunidad y en su interés personal de vivir de manera más saludable; asimismo, en lograr relaciones más solidarias para que la comunidad tenga más atención de la política pública para el acceso a servicios de salud, educación, entretenimiento y otros derechos inherentes al desarrollo humano.

Los factores de conversión personal e institucional arrojan información clave para los equipos técnicos que desarrollan los procesos. La cultura de poca participación y cohesión social, el temor a la toma de decisiones y las jornadas amplias de trabajo en las fincas son parte de las barreras que impiden a las personas aprovechar los procesos de formación, gestar el desarrollo comunitario y realizar proyectos asociativos. Las personas locales piden un mayor compromiso y 
dedicación de los técnicos o consultores que realizan los procesos formativos y el acompañamiento, así como la planificación de iniciativas que garanticen procesos con una visión y permanencia en terreno de largo plazo. Desde sus aspiraciones, las personas esperan obtener resultados en un menor plazo y mayor acompañamiento en las distintas fases del proyecto, por lo que también se requiere de mayor presencia y efectividad de las acciones desarrolladas. Los procesos de desarrollo rural, además, debe apoyar a la comunidad en la búsqueda de recursos financieros no reembolsables y/o créditos blandos para lograr aplicar los conocimientos adquiridos e inversiones en infraestructura y equipo. También, claman por apoyo en la generación de alianzas con otras organizaciones que les permita sostener las iniciativas a futuro y generar nuevos proyectos locales que beneficien a sus comunidades y a sus familias.

\section{Bibliografía}

Alkire, S. (2002). Dimensions of human development. World Development, Vol. 30. Printed in Great Britain.

Ayllon Pino, B., Ojeda Medina, T., \& Bancet, A. (2013). La cooperación Sur-Sur en la gobernanza del desarrollo: nuevas configuraciones de la arquitectura de la ayuda. Serie Documentos de Trabajo. Madrid: Instituto Universitario de Desarrollo y Cooperación IUDC-UCM.

ASOLVI. (2017). Boletín Informativo Asociación Linda Vista de Colorado (ASOLIVI). Limón, Costa Rica.

Bermúdez, F; Chuprine, A. (2009). Ministerio de Ambiente, Energía y Telecomunicaciones. Sistema Nacional de Áreas de Conservación. Plan de Manejo de Refugio Nacional de Vida Silvestre Barra del Colorado. Pococí, Limón, Costa Rica.

Boni, A., Calabuig, C., Cuesta, I., de los Llanos, M., Lozano, J. F., Monzó, J. M., \& Torres, A. J. (2010). La cooperación internacional para el desarrollo. Universitat politècnica de València.

Calvo, S; Marín, E. (2014). Universidad Nacional de Costa Rica. Análisis de la incidencia del Programa de Gestión Local y Corredores Biológicos en el desarrollo sustentable de las comunidades de Linda Vista y la Aldea, Refugio Nacional de Vida Silvestre Barra del Colorado. Costa Rica.

Haq, M. (1995). Reflections on human development. United States. Oxford University Press. 
Inge Kaul, I. G.; Stern, M. A. (2001). Bienes públicos mundiales: la cooperación internacional en el siglo XXI. México, DF.

Unceta, K.; Yoldi, P. (2000). La cooperación al desarrollo: surgimiento y evolución histórica. Vitoria-Gasteiz: Servicio Central de Publicaciones del Gobierno Vasco.

López, M; Lizano, M; Salas, R. (2016). Informe final de consultoría. Facilitación para la identificación de potencialidades productivas y mercadeo de los productos del sistema de fincas integrales en el refugio Nacional de Vida Silvestre Barra del Colorado (II Fase). Costa Rica.

Ministerio de Planificación Nacional y Política Económica (MIDEPLAN). (2014). Sistema de Gestión de la Cooperación Internacional (SIGECI). San José, Costa Rica.

Ministerio de Planificación Nacional y Política Económica (MIDEPLAN). (2014a). Plan Nacional de Desarrollo 2015-2018 “Alberto Cañas Escalante”. San José, Costa Rica.

Ministerio de Planificación Nacional y Política Económica (MIDEPLAN). (2014b). Sistema de Gestión de la Cooperación Internacional (SIGECI). San José, Costa Rica.

Ministerio de Planificación Nacional y Política Económica (MIDEPLAN). (2017). Índice de Desarrollo Social. San José, Costa Rica.

Montes, R y Matamoros Y. (2011). Diagnóstico Participativo del distrito de Llanuras del Gaspar. Universidad Nacional (UNA), Costa Rica.

Montúfar, César. (2001). Hacia un nuevo marco interpretativo de la asistencia internacional para el desarrollo. Comentario Internacional: Revista del Centro Andino de Estudios Internacionales (Quito), 173-188.

Munguía Y. y Granados R. (2012). Diagnóstico del cantón de Pococí. (2012). Ministerio de Vivienda y Asentamientos Humanos. República de Costa Rica.

PNUD. (1990). Informe desarrollo humano 1990. Programa de las Naciones Unidas Para el Desarrollo. Pub. PNUD-Tercer Mundo Edics. Bogotá. Colombia.

PNUD. (2017). Informe Nacional de Desarrollo Humano. Desarrollo humano. Guatemala. Disponible en: http://desarrollohumano.org.gt/desarrollo-humano/concepto/Revisado: 01 de abril de 2017.

Robeyns, I. (2003). The capability approach: an interdisciplinary introduction, teaching material for the raining course preceding the 3rd International Conference on the capability approach. Pavia, September. 
Sanahuja, J. A. (2005). Seguridad, desarrollo y lucha contra la pobreza tras el 11-S: los Objetivos del Milenio y la "securitización" de la ayuda". Documentación social, 136, 24-41.

Sen, A. (2000). Libertad y desarrollo. Bogotá: Editorial Planeta.

Sogge, David. (2009). Sistema de ayuda extranjera:¿ Régimen o vehículo hegemónico?| The Foreign Aid System: Regime or Hegemonic Vehicle?. Relaciones Internacionales, (12).

Walker, M. (2014). Universidades, desarrollo humano y justicia social: el enfoque de capacidades. En Universidad y Cooperación al Desarrollo. Estudios del Desarrollo y Cooperación Internacional. Universidad Politécnica de Valencia. España.

Denzin y Lincoln (2005). Manual de investigación cualitativas. Editorial Gedisa, Vol. I.

\section{Agradecimientos}

Se agradece profundamente a los actores locales por la dedicación, la apertura y el tiempo entregado, que permitieron acceder a sus experiencias y vivencias en torno a las iniciativas de cooperación en sus comunidades. Asimismo, a las contribuciones de los funcionarios de SINAC y MAPCOBIO, entre ellos a Luis Rojas, Aurora Camacho, Mariana Jiménez, Erick Herrera, Sonia Calvo y Lorelly Soto. A la Escuela de Planificación y Promoción Social de la Universidad Nacional (UNA) y al Centro de Estudios Generales (CEG), que aportaron recursos para la recolección de datos y el trabajo de campo, así como a las estudiantes Estefanía Fernández y Fiorella Barrantes que apoyaron durante ese proceso. Las y los autores hacen un especial reconocimiento a la doctora Maritza González-Moreno de la CUJAE, Cuba, por su lectura y observaciones ofrecidas al documento. 